\title{
Crónica
}

\section{MEMORIA DEL PRESIDENTE DE LA SOCIEDAD CHILENA DE OBSTETRICIA Y GINECOLOGÍA, 2006 - 2007}

Estimados colegas: hoy es un día muy especial para mí, las emociones y sentimientos se agolpan ante mis ojos y no es fácil expresarlos. Después de 16 años al servicio de nuestra querida Institución y habiendo ocupado todos los cargos del directorio, incluso uno de ellos en dos oportunidades, puedo decir sin duda alguna misión cumplida. También puedo contarles que en estos años he vivido momentos de gloria y alegrías de nuestra institución, pero a si mismo momentos amargos y agrios. Sin embargo, todos ellos nos han hecho crecer y madurar. He aprendido que cada directorio tiene su propio afán y surgen los liderazgos que iluminan el camino, vaya mi agradecimiento a cada uno de ellos que fueron mi escuela y por qué no decirles mi espejo donde consultar en los momentos de decisiones.

Debo solicitarles se pongan de pie y guardemos un minuto de silencio porque hemos perdidos hijos predilectos en este período, que fueron ejemplos de vida y me refiero a la Dra. Silvia Segovia y al Dr. Raúl Saavedra. Ambos trabajaron incesantemente por nuestra institución llegando a ser presidentes, miembros honorarios, maestros chilenos y latinoamericanos de Obstetricia y Ginecología, es más la Dra. Segovia era nuestra candidata al premio Nacional de Medicina. Sus ejemplo de nobleza y entrega nos compromete a ser humildes, agradecidos y a ser faros que iluminen las generaciones venideras.

Quisiera ahora relatarles algunos de nuestros logros y dificultades en el ejercicio de este periodo 2006-2007:

1. Reuniones científicas. Enfrentamos el primer gran escollo, el lugar físico. La Clínica Alemana entró en remodelación y ampliación de su planta física motivo por el cual durante el 2006 y prácticamente la mitad del 2007 tuvimos que sesionar en el auditorio del Laboratorio Saval, estamos muy agradecidos pero esto significó una merma en la asistencia por ese solo hecho y también por lo limitado del espacio, la costumbre y tradición de tantos años se rompió. Sin embargo, realizamos las siguientes reuniones científicas: en 2006, 12 sesiones científicas y una sesión extraordinaria y en 2007,14 sesiones científicas. Las reuniones de Directorio se realizaron en nuestra sede de Román Díaz. Como la caridad empieza por casa, en este período pintamos y cambiamos el piso quedando una sede más moderna y acogedora.

2. Reuniones de Directorio. Se realizaron 14 reuniones en 2006 y 18 reuniones en 2007.

3. Revista Chilena de Obstetricia y Ginecología. También hemos sufridos cambios drásticos y dramáticos. El Dr. Enrique Donoso, Editor Jefe, ha hecho una labor encomiable que merece toda nuestra gratitud. La revista en su versión electrónica se encuentra al día, pero su presencia física se ha visto dificultada por la falta de respuesta a tiempo del que fue nuestra imprenta por tantos años, motivo por el cual tuvimos que tomar la lamentable decisión de cambiarla con todas las consecuencias que de ello deriva. Además, recibimos un cambio sustancial en el financiamiento. Agradecemos a las casas farmaceúticas que aún nos apoyan. Esto ha hecho meditar profundamente la necesidad de cambiar la manera de financiar nuestra revista para el futuro. Tarea del próximo directorio.

4. Página Web. Recibimos una página Web acorde con los tiempos, pero los tiempos cambian y tuvimos la necesidad de mejorarla, haciéndola mas activa, dinámica y moderna, hoy es motivo de elogios a nivel nacional e internacional, con una gran cantidad de visitas. Es un instrumento de comunicación, difusión, educación e intercambios de opiniones entre otros, tanto así que muchas instituciones científicas, académicas y gremiales nos solicitan les hagamos difusión de sus actividades esenciales. De nuestros países vecinos es habitual que nos pidan la difusión de sus eventos y congresos.

5. Ingreso de nuevos socios. Hemos tenido una cantidad record de ingresos y son los siguientes Drs: 
Rodrigo Latorre, José Antonio Arraztoa, Carolina Schulin-Zeuten, Susana Aguilera, Sócrates Aedo, Alberto Selman, Macarena Socias, Julio Astudillo, Mauro Parra, Guillermo Durruty, Manuel Donoso, Sebastián Illanes, Pablo Torres y Carlos Wenzel.

6. Red Informática de Medicina Avanzada (RIMA). Un hecho relevante ha sido el logro de contar para todos los socios con RIMA. Ha sido el gran aporte del Laboratorio Farmacéutico Wyeth. El solo acceso gratuito a la red mas grande de bibliotecas, revistas nacionales e internacionales, metaanálisis, opiniones de expertos, en forma eficiente, rápida y efectiva, nos permiten cubrir el rol de educación continua mas trascendente de los últimos tiempos. 7. Relaciones con Sociedades satélites. Prometimos intentar acoger y acercar todas las sociedades satélites de nuestra especialidad bajo el alero de SOCHOG, tuvimos algunas reuniones de directorio para invitarlos a participar en algunas de nuestras actividades científicas, es así que tuvimos una notable Jornada de Obstetricia y Ginecología, en Santiago, en Noviembre de 2006. En esa oportunidad tuvimos módulos a cargo de las Sociedades de Ginecología Infanto-Juvenil, de la Sociedad de Andrología, de la sociedad de Climaterio y Menopausia, de la sociedad de Oncología Ginecológica. Siendo desde el punto de vista académico muy exitoso, muy fraternal y financieramente positivo, dignas de ser repetidas. No es aconsejable atomizarnos en sociedades pequeñas, que lo único que logran es duplicar esfuerzos de todo tipo. Aunque sea políticamente incorrecto debemos hacer un llamado a todos los socios y no socios, ya que somos los mismos y pocos, ha abrir nuestra Sociedad a la participación activa de todos, si es necesarios cambiar estatutos, lo haremos. Solo de esta manera dejaremos de ser una elite cuya fuerza inexorablemente se debilitara en el tiempo, por un problema de masa crítica.

8. Relaciones internacionales. Hemos tenido un tremendo respaldo internacional. Chile realizó un hermoso y fantástico Congreso mundial de la especialidad en 2003, que aún tiene ecos a nivel nacional y en el extranjero. Sin embargo, las ambiciones de personas y regionales, a mi modesta opinión, quisieron relegar el legado de Chile a algo del pasado. Por este motivo tuvimos que trabajar arduamente para lograr durante el Congreso Mundial en Kuala Lumpur, Malasia. Seguir siendo miembro del Executive Board de FIGO, hasta el año 2012. Fue necesario convencer a Canadá, EEUU, Japón y otros, que nos otorgaron sus votos para lograr esta meta. FIGO cuenta con 114 países miembros, cada país tiene derecho a un voto cada 500 socios hasta un máximo de 5 . Chile tiene apenas un voto y los miembros del Executive Board son solo 24 países, por lo tanto ustedes comprenderán este logro significativo. Desgraciadamente, Latinoamérica nos dio la espalda ya que nuestros intereses no concordaron, por esto es tarea prioritaria trabajar para mejorar los lazos y nexos con nuestros hermanos de la Región. Nuestra gran misión es trabajar para representar a Chile y Latinoamérica de la mejor manera posible. También el apoyo recibido compromete nuestra lealtad si pretendemos conservar esta situación de privilegio.

9. Sección Chilena del Colegio Americano de Obstetras y Ginecólogos. Hito de gran importancia ha sido la formación de la única sección del Colegio Americano en Sudamérica. Esto significa un gran reconocimiento a la formación de especialistas de nuestro país, y a la calidad de la educación de pre y postgrado de nuestras Universidades. Ha sido un camino largo, de mucho trabajo silencioso pero que han dado sus frutos. A partir del $1^{\circ}$ de Julio de 2007 se nominaron los primeros socios fundadores del capitulo chileno, me otorgaron el honor, el privilegio y una gran responsabilidad de proponer los nombres de cada uno de ellos, siendo requisito ser socios de SOCHOG. Se eligieron pensando en razones de méritos personales, liderazgos, cualidades humanas, conveniencias estratégicas para los directivos de SOCHOG, etc. y por cierto por la limitación de un número razonable. Afortunadamente ninguno de los propuestos fue rechazado y se me permitió nombrar algunos más que abarcaran otros ámbitos y criterios. Durante el XXXI Congreso Chileno de Obstetricia y Ginecología, se formalizó la formación de la Sección Chilena de ACOG, siendo nominado el suscrito como el primer Presidente de esta Institución y Vicepresidente el Dr Eugenio Suárez. En Mayo de 2008, en New Orleans, Louisiana, EEUU, todos los fellows fundadores serán investidos en una ceremonia muy hermosa. Esta nueva institucionalidad será totalmente bajo el alero de SOCHOG, no se pretende formar instituciones paralelas, ni competitivas entre si. Tanto así que en noviembre de este año realizaremos el primer Congreso Chileno de la Sección Americana de ACOG, en vez de las Jornadas que estábamos acostumbrados a realizar. En este evento se nominaron los primero miembros de este capitulo. La trascendencia es de un valor incalculable, sobretodo en el gran aporte en educación continua para nuestros socios, y hemos abierto un portal para la posibilidad de formación en Norteamérica de nuestros socios y para todos los médicos en formación en nuestra especialidad. Tal vez esto nos permita acoger en parte a las sociedades satélites, que tendrían una oportunidad única de participación. 
Además quiero destacar la alianza que hemos pactado con la Sociedad Canadiense de Obstetricia y Ginecología, que durará al menos 5 años, es tarea de todos sacarle el máximo provecho.

10. XXXI Congreso Chileno de Obstetricia y Ginecología. Realizado en la ciudad de Viña del Mar, en el mes de Noviembre de 2007. En este Congreso en particular, decidimos instaurar algunas tradiciones, emblemas y signos, manteniendo las tradiciones pero innovando. Durante la cena del directorio se homenajearon a todos los past presidente vivos de nuestra Sociedad, entregándoles un diploma de agradecimiento por sus presidencias y una insignia de oro con el emblema de la Venus de Milo y que los distinguirá en los futuros congresos como past presidente. Es increíble lo frágil de la memoria y muchas veces las nuevas generaciones desconocen quienes dieron todo durante muchos años, hemos querido corregir esta falencia. Me llena de alegría el haber visto la emoción en los ojos y en el corazón de nuestros presidentes en el momento del reconocimiento, hoy algunos ya nos han dejado con su legado. Quisimos también reconocer como maestros chilenos en Obstetricia y Ginecología a tres grandes personas, me refiero a los Drs Alfredo Pérez, Patricio Vela y Pablo Escudero, sus méritos hablan por si solo. Se dieron los títulos de Miembros Honorarios a algunos de nuestros past presidentes: Drs Alfredo Saumann y Cristián Miranda, también se nominaron miembros honorarios extranjeros a los Drs Dorothy Shaw Presidente actual de FIGO, Kennet Noller Presidente de ACOG, Ralph Hale Vicepresidente de ACOG, Andre Lalonde Vicepresidente de SOGC, Neville Hacker, y Oscar Contreras. Una ceremonia inolvidable para este humilde servidor. EI XXXI Congreso Chileno contó con una cifra record de 600 médicos de la especialidad, lo que habla muy bien de calidad de éste, por primera vez se cambió la modalidad de participación, habiendo una gran cantidad de cursos transcongresos que abarcaron prácticamente todo el quehacer gineco-obstétrico y más. Tuvimos El Curso ALARM (Advances in Labour and Risk Management) en conjunto con la Sociedad Canadiense de Obstetricia y Ginecología, participaron 47 médicos que se sometieron a rigurosos exámenes escritos y orales que le permitieron aprobar al menos al $75 \%$ de ellos. Este examen es requisito fundamental para la recertificación de la especialidad cada 5 años en Canadá, vaya a todos ellos mis felicitaciones incluso aquellos que no aprobaron ya que tiene la excelente oportunidad de mejorar en el futuro. Esto permitirá contar con un sinnúmero de monitores que podrán ir en ayuda de nuestros países vecinos y mejorar la calidad de vida de las mujeres. La asis- tencia por primera vez en Chile de la presidenta de FIGO, la plana mayor de ACOG y la presencia de la Sociedad Canadiense con Dr. Lalonde a la cabeza, habla de un respaldo internacional hacia nuestra institución, por decir lo menos, óptima. Debemos cultivar y mantener esta confianza.

11. Otras actividades. En el plano Latinoamericano hemos participado en todas las actividades de FLASOG, lealmente con un bajo perfil, para no despertar anticuerpos en nuestros hermanos, desde lugares secundarios hemos hecho nuestro aporte en beneficio de la amistad y la concordia, y lo seguiremos haciendo. En el ámbito nacional hemos participado en numerosas actividades, las mas importantes tal vez han sido la cooperación con el Ministerio de Salud en quehacer diarios, nos hemos hecho participes en la confección de manual de las normas del manejo actual del embarazo y parto. Hemos dado un respaldo a la necesidad de legislar para toda la población el acceso gratuito a los métodos de planificación familiar, pero haciendo hincapié en la necesidad de planes de educación sexual a todos los niveles aconsejables. El Directorio ha sido muy cauto y prudente, tomando en consideración el equilibrio en todas las manifestaciones valóricas y muy democrático a la hora de expresarse, pero siempre velando por el interés común y por mejorar la salud de nuestras mujeres. Seria muy largo relatar todas la actividades que hemos respaldados a través de ASOCIMED, que se ha transformado en un actor importante junto a ASOFAMECH, Colegio Médico, Academia de Medicina y CONACEM, por velar por la certificación de nuestras especialidades y subespecialidades y por la acreditación de nuestros centros de acreditación de pre y postgrado. Quisiera agradecer a todos los socios que nos representan en estas entidades. Durante los dos años otorgamos auspicio a varios actividades científicas nacionales relacionadas con la especialidad y que cumplían con los requisitos exigidos por nuestra institución para otorgar el auspicio. En este período también presentamos a la Asamblea algunas modificaciones en beneficio de la institucionalidad, es así que contaremos en el futuro con la figura de Past President, el que podrá participar en forma voluntaria en la reuniones de Directorio, con derecho a voz pero no a voto, lo que permitirá sacar provecho de la experiencia adquirida durante el ejercicio del cargo. También se creo la figura del Presidente Electo que corresponde al cargo del Vicepresidente actual; además la Sociedad podrá considerar miembros aliados a una serie de instituciones que no tengan fines de lucro que tengan como meta mejorar la salud y la calidad de vida de nuestras mujeres. Es necesario en el fu- 
turo profundizar aun mas los cambios, debiéramos abrir la Sociedad a otros profesionales de la salud, como miembros asociados siguiendo el ejemplo de la gran mayoría de las instituciones de prestigio internacional. Tal vez la reflexión mas importante acerca del quehacer del Directorio es conseguir la profesionalización de la infraestructura que soporta nuestras actividades, es probable involucrar profesionales con experiencia en el marco de negocio y marketing. Otra actividad de vital importancia fue poner en marcha por primera vez el Comité de Búsqueda de profesionales para la formación del nuevo Directorio, tarea de suyo interesante, motivante y de gran responsabilidad que requirió de varias horas de trabajo para llegar a un consenso unánime, tanto así que el Directorio recientemente electo fue elegido por aclamación en cada uno de sus cargos, sin objeción alguna por parte de la Asamblea. La Sociedad es y debe ser un gran actor en quehacer académico, científico, cultural, social y gremial de nuestro país a través de los distintos canales de participación. No tengo duda que hoy ocupamos un espacio preponderante siendo interlocutores válidos para las autoridades ministeriales, judiciales, parlamentarias, etc. y que a menudo nos consultan y nos hacen participes de decisiones importantes para nuestra población. He tratado de no cometer el error de nombrar personas, pero siempre es menester ser agradecido. Cada una de ellas sabe con acciones lo valioso que han sido para mí. He aprendido de personas y personajes en el transcurso de tantos años, de todas he tratado de sacar lo más útil y provechoso, de destacar las virtudes de ellas, créanme que no siempre es fácil y bonito. Sin embargo, la suma de éxitos y fracasos hoy me permiten decir: "SOCHOG, tanto me has dado, tanto te di, estamos en paz".

Muchas gracias,

Dr Eghon Guzmán B. Past President, 2006 - 2007. 